\title{
Nurse Dose: What's in a Concept?
}

\author{
Milisa Manojlovich, ${ }^{1} *$ Souraya Sidani ${ }^{2} * *$ \\ ${ }^{1}$ University of Michigan School of Nursing, 400 N. Ingalls, Room 4306, Ann Arbor, MI \\ ${ }^{2}$ Ryerson University School of Nursing, Toronto, Ontario, Canada \\ Accepted 2 October 2007
}

\begin{abstract}
Many researchers have sought to address the relationship between nursing care and patient outcomes, with inconsistent and contradictory findings. We conducted a concept analysis and concept derivation, basing our work on theoretical and empirical literature, to derive nurse dose as a concept that pulls into a coherent whole disparate variables used in staffing studies. We defined nurse dose as the level of nursing reflected in the purity, amount, frequency, and duration of nursing care needed to produce favorable outcomes. All four parameters of nurse dose used together can facilitate our understanding of how nursing contributes to patient outcomes. Ongoing investigation will help to identify the parameters of nurse dose that have the greatest effect on outcomes. () 2008 Wiley Periodicals, Inc. Res Nurs Health $31: 310-319,2008$
\end{abstract}

Keywords: concept analysis; concept derivation; theory building; nurse staffing

Understanding how nursing care as an intervention contributes to good outcomes for hospitalized patients is still unclear despite a vast number of studies on the subject. The atheoretical nature of much of the staffing research (Mark, Hughes, \& Jones, 2004; Verran, 1997), as well as inconsistent and contradictory findings across studies (Jiang, Stocks, \& Wong, 2006) have precluded efforts to link staffing variables to patient outcomes. Consequently, the purpose of this article is to begin building a theoretical base from which to understand the link between nursing care and patient outcomes. We describe the results of a concept analysis and a concept derivation undertaken to clarify the conceptual and operational definitions of nurse dose, a concept underlying staffing research and guiding measurement of nursing's contribution to patient care.

Nurse dose is not a new term. Nurse dose was first introduced as a term to reflect the number of nurses and the skill mix needed to demonstrate the contribution of nursing to patient outcomes (Brooten \& Naylor, 1995). Brooten and Youngblut (2006) reconceptualized nurse dose as having three components: the number of nurses, the amount of time spent with patients, and the number of patient contacts. The conceptualization proposed by Brooten and Youngblut represents an evolution in nurse dose, and hints at its potential use in nursing staffing research. However, in distinguishing between what constitutes a dose and what reflects a nurse, this conceptualization does not conform to the scientific definition of the concept dose, which we discuss later in this article. Further, it does not provide an explicit conceptual definition of nurse dose. We agree with Brooten and Youngblut in their acknowledgement that nurse dose requires clarification as a concept.

In this article, we clarify the conceptualization of nurse dose by identifying its critical attributes

We are grateful to the Associate Editor and anonymous reviewers for constructive feedback and insightful suggestions, which greatly improved this article.

Correspondence to Milisa Manoilovich

*Assistant Professor.

**Professor.

Published online 29 January 2008 in Wiley InterScience

(www.interscience.wiley.com) DOI: 10.1002/nur.20265 
and empirical indicators. We adapted an explicit, organized approach to theory construction, blended with our own intuitive processes, as suggested by Walker and Avant (2005). The strategies of concept analysis and concept derivation served as guideposts on our journey to conceptualize variables used in staffing studies as parts of a greater whole.

We began by analyzing the concept dose itself, to clarify the conceptualization and operationalization of dose that are commonly used when describing interventions. We were able to identify and define the empirical indicators of dose as described in behavioral and health-related disciplines. We then used the results of concept analysis to conduct a concept derivation. We reviewed relevant nursing literature to derive a new meaning for dose, within the context of acute care nursing, ultimately constructing a theoretically coherent concept called nurse dose. By clarifying the conceptual and operational definitions of nurse dose, we offer a unified view of the inter-relationships among staffing variables, with the goal of advancing research on nurse staffing and patient outcomes.

\section{DOSE: CONCEPT ANALYSIS}

The purpose of the concept analysis was to identify the critical attributes and empirical indicators that operationally define the concept of dose, thereby clarifying it. Critical attributes are characteristics of a concept that indicate the presence of that concept. Empirical indicators arise from the critical attributes and become specific variables that are used to measure the critical attributes. The concept analysis was conducted in several steps.

First, an extensive search of the healthcare literature was conducted to find as many conceptual and operational definitions as possible of dose. Relevant literature from different disciplines was searched, including medicine, behavioral, and health-related sciences, because the concept of dose has rarely been used in nursing. The following databases were searched: CINAHL, ERIC, and PSYCLIT, using the keywords of "dose" and "dosage" combined with "treatment(s)" or "intervention(s)."

Second, published theoretical and empirical articles were selected for review. We included published theoretical articles if they provided conceptual and operational definitions of dose. These articles $(n=4)$ were reviewed to identify critical attributes reflective of dose. Attributes of dose were then compared. In the process it became evident that there were more similarities in critical attributes than differences across all theoretical papers. Empirical articles $(n=10)$ consisted of reports of program or intervention evaluation studies. They were included in the review if they measured the dose of the intervention under evaluation. The way in which intervention dose was operationalized was critically appraised to determine empirical indicators of dose.

Third, the operationalization of dose in empirical articles was examined for its consistency with the attributes identified in the theoretical papers. In all cases, the operationalization of dose was consistent with the attributes defining this concept, lending empirical support for the critical attributes and empirical indicators of dose. The results of concept analysis are presented next.

In health-related disciplines other than nursing, the most common meaning of dose is the amount of a treatment or intervention, whether in the form of drugs or other therapeutic agents such as radiation or laser beams (Scott \& Sechrest, 1989). In behavioral and health-related literature, dose is discussed in relation to the entire course of an intervention (Scott \& Sechrest) and generally refers to the level of treatment (Lipsey, 1990). In all disciplines, dose of a treatment or intervention is viewed as a multi-dimensional concept that can not be adequately operationalized by one critical attribute.

The four critical attributes of dose that emerged through the concept analysis included: purity, amount, frequency, and duration. Purity refers to the concentration of the active elements of a treatment. Amount represents the quantity with which the active elements are to be given. Frequency refers to the number of times the active elements are to be given over a specified period of time. Duration is the total length of time during which the active elements of a treatment are to be given.

Thus, dose is a function of many attributes that, taken together, determine the level of the treatment needed to produce the intended outcomes (Lipsey, 1990). For instance, a medication dose stated as $300 \mathrm{mg}$ or a behavioral intervention dose described as two sessions is insufficient information for prescribing the dose to patients. Information on frequency (how often) and duration (for how long) are also needed. A specification of all attributes of dose is necessary to guide treatment delivery. Table 1 summarizes critical attributes of dose, providing literature sources for each attribute, definitions, and examples.

We followed Walker and Avant's (2005) recommendation to identify both irrelevant attributes and 


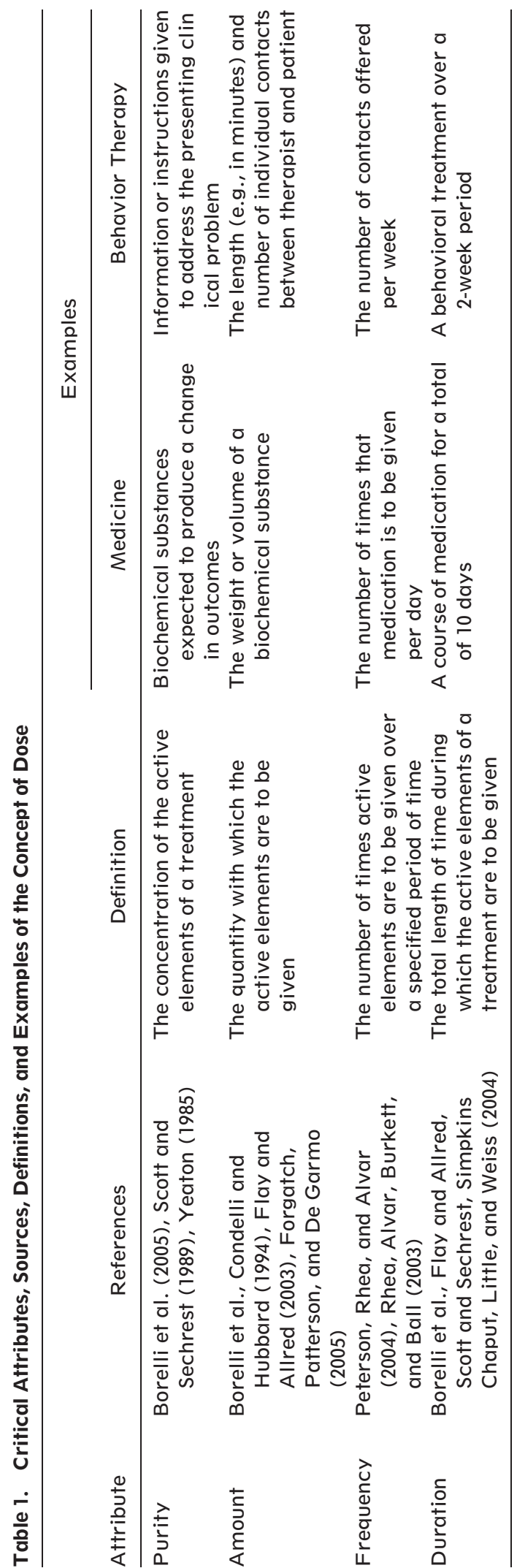

related concepts as part of the concept analysis. In this analysis, it became evident that the term strength has been used interchangeably with dose. In-depth analysis indicated that the two terms are distinct, however. Dose refers to the level at which the treatment is to be given for the intended outcomes to take place, whereas strength is the magnitude of the response to the treatment capturing treatment effectiveness in producing the outcomes (Lipsey, 1990). Dose is therefore an antecedent to strength, while strength is a consequence of dose. For example, two patients experiencing pain are administered the same analgesic, but at different doses. One patient receives a $300 \mathrm{mg}$ dose, while the other receives a $400 \mathrm{mg}$ dose. Both patients later report an identical decrease in pain, as measured by a numeric rating scale. Although the doses differed, the strength (the magnitude of the response) was the same.

Dose is always discussed in relation to the treatment or intervention under evaluation; it represents one element that is essential for an appropriate implementation of the intervention. This point emphasized for us the importance of considering nurse dose as a unified concept and not as separate components (i.e., nurse and dose). We were encouraged by the fact that we were able to find critical attributes and empirical indicators of dose through concept analysis. The search for conceptually congruent critical attributes and empirical indicators of nurse dose was guided by our conviction that nurse dose needed a theoretical foundation. The steps of concept derivation as described by Walker and Avant (2005) provided a meaningful and creative approach for our search.

\section{NURSE DOSE: CONCEPT DERIVATION}

The purpose of the concept derivation portion of our theory building endeavor was to clarify the operationalization of nurse dose, which is conceived as a new concept underlying staffing research. Concept derivation allows a concept in one field to be redefined in another, creating a new concept, thereby contributing to advancement in the latter field (Walker \& Avant, 2005). The conceptualization and operationalization of nurse dose were derived through an analogy and correspondence analysis between dose and nurse dose. Our strategy for conducting concept derivation consisted of several steps.

First, the critical attributes of the concept of dose served as the foundation for the concept derivation exercise. We were inspired by the notion of dose as 
described in medicine, behavioral, and healthrelated disciplines, where a multi-dimensional conceptualization of dose was presented, and contained in four critical attributes (i.e., purity, amount, frequency, duration). This conceptualization of dose held promise because it allowed parallels to variables used in staffing studies to be pulled together into a comprehensive set of critical attributes and empirical indicators reflecting the concept of nurse dose.

The second step of concept derivation involved a review of pertinent nursing research to identify commonly investigated staffing variables and their corresponding specific empirical indicators. We used the keywords of "staffing" and "skill mix" combined with "outcomes," limiting our search to one database, CINAHL, which pertains to nursing. Although we found very little on nurse dose in the nursing literature, the review deepened our understanding of the staffing literature and facilitated the correspondence analysis. It also helped us determine that future staffing research could be strengthened by the concept of nurse dose. Research studies published in English were included in the review if the focus was on the examination of relationships between staffing variables and outcomes of nursing care for hospitalized patients. Seventeen studies met the inclusion criteria for review. Data on the conceptual and operational indicators of staffing variables, and the direction of their relationships with outcomes were abstracted. Table 2 presents data abstracted from a representative sample of staffing studies used to determine the empirical indicators for the critical attributes of nurse dose.

The third step of concept derivation encompassed an analysis of the indicators of dose and staffing variables in an attempt to determine conceptually meaningful correspondence among them. Similar to the concept of dose, we found that nurse dose could be conceived of as a multidimensional concept that is operationalized in several equally important critical attributes.

In the fourth and final step we redefined the concept of dose and its attributes in terms of nurse dose and relevant empirical indicators identified from staffing research. We defined nurse dose as the level of nursing reflected in the purity, amount, frequency, and duration of nursing that is needed to produce favorable outcomes. We included both registered nurses (RNs) and licensed practical nurses (LPNs) in nurse dose, since both types of nurses were included in staffing studies.

The critical attributes of nurse dose, by definition, are inter-related as they reflect a common concept. As with any other concept, assessment of all critical attributes is necessary to accurately represent nurse dose. The empirical literature identified linear relationships among the critical attributes and empirical indicators of dose and outcomes; non-linear relationships should be explored as well. Table 3 summarizes results of the concept derivation process.

\section{Nurse Dose: Purity}

In pharmacology purity refers to the concentration of the active element of a particular drug or treatment. Purity is one of four critical attributes of dose, and the concentration of the active element is the empirical indicator of purity. To develop an analogous notion of nurse dose purity, we had to determine what the active element of nursing would be and then decide how the active element could be converted into an empirical indicator. We believe that nursing knowledge is the active element of nursing, and that nursing knowledge can be used to operationalize nurse dose purity. Instead of just one empirical indicator, we determined that three empirical indicators are needed to fully depict the concentration of nursing knowledge: nurses' education, experience, and skill mix.

Nurse education refers to formal schooling leading to the granting of a nursing degree (associate through doctoral degree), or diploma (licensed practical nurse or nursing diploma program). Higher academic degrees in nursing are associated with more nursing knowledge. The association of nursing education to outcome variables has been mixed, with some researchers reporting a positive relationship between education and outcomes (Aiken, Clarke, Cheung, Sloane, \& Silber, 2003; Estabrooks, Midodzi, Cummings, Ricker, \& Giovanetti, 2005), while others have been unable to demonstrate a link between the two (Blegen, Vaughn, \& Goode, 2001).

Nurse experience is defined as the number of years in direct nursing practice. Through direct interactions with patients, experienced nurses have gained more knowledge that is transferable to the care of various patient populations than novice nurses. Blegen et al. (2001) found that on nursing units with more experienced nurses, there were fewer medication errors and fewer patient falls, suggesting a relationship between experience and patient outcomes. Education and experience were selected as indicators of purity because nurses with high levels of education and extensive clinical experience should have 


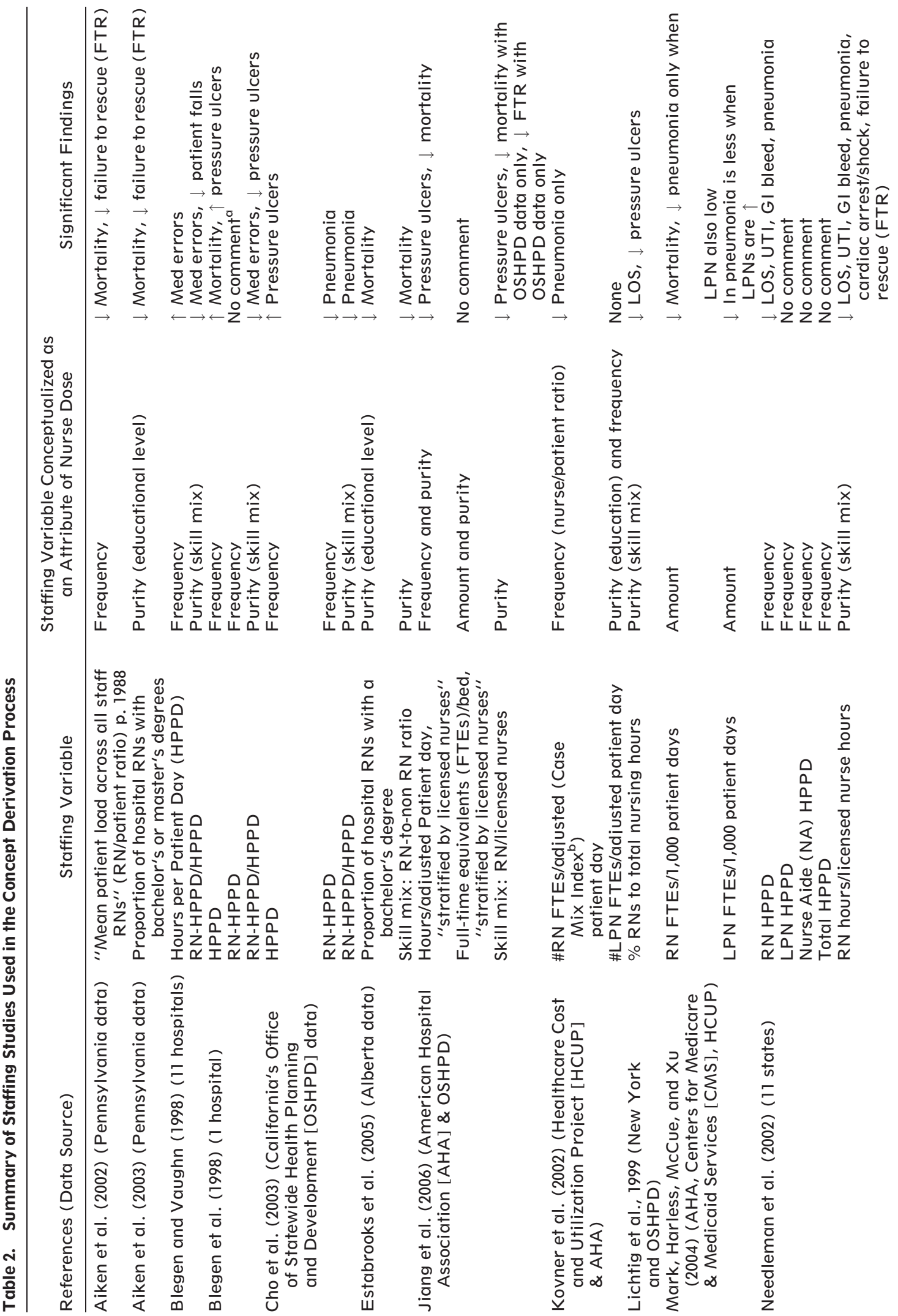



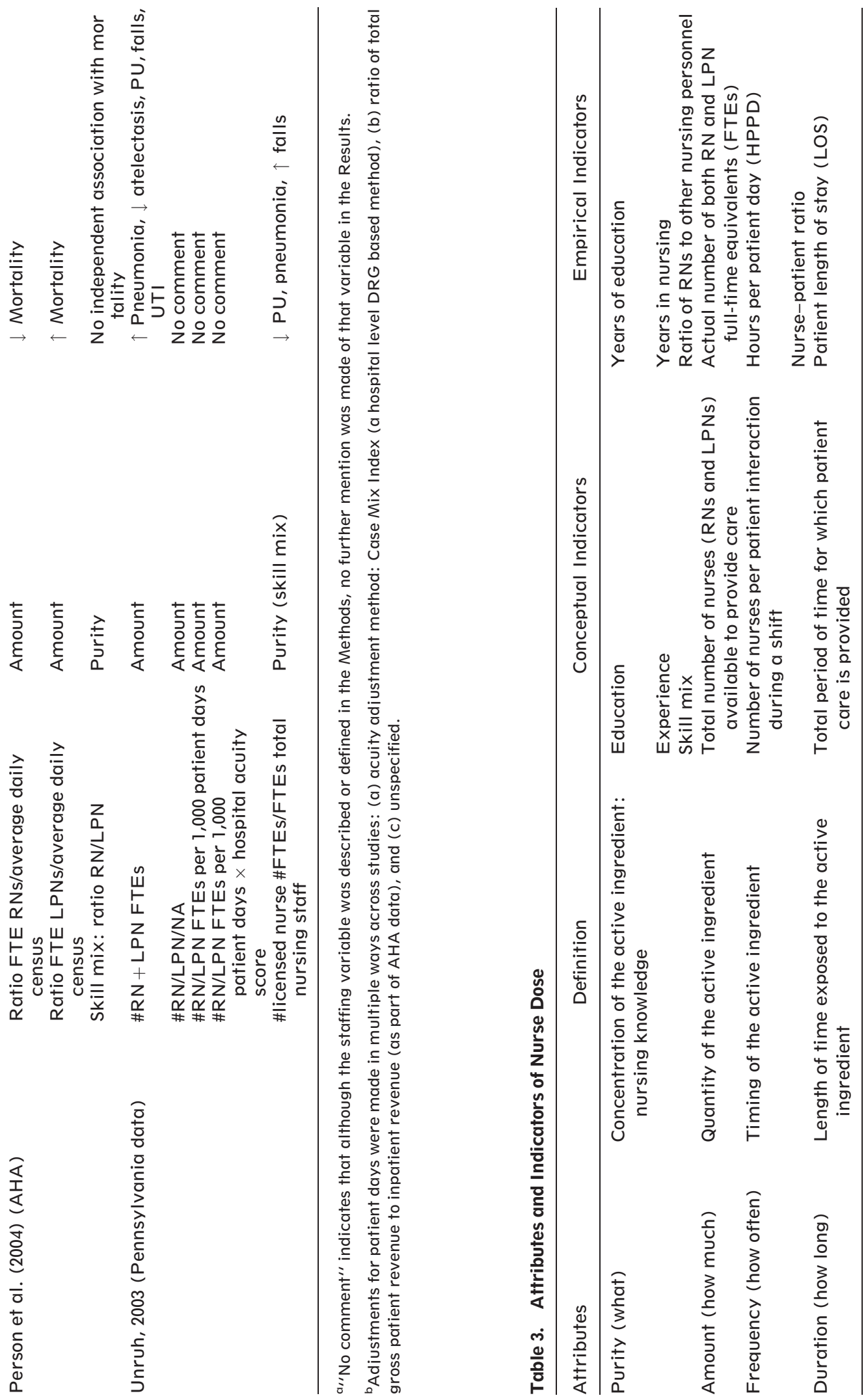
gained the theoretical and practical knowledge required for the provision of high quality nursing care.

Skill mix, sometimes referred to as staffing mix, is defined as the ratio of registered nurses (RNs) to other nursing personnel. A richer skill mix is associated with a higher proportion of RNs, who have had more formal educational preparation in nursing knowledge than non-RN staff (American Academy of Colleges of Nursing, 2001; Boyce et al., 2001). Unruh (2003), Person et al. (2004), and Jiang et al. (2006), found that a skill mix characterized with a high proportion of RNs was associated with lower adverse outcome rates.

Nurse staffing literature includes investigations of education, experience, and skill mix, but not all in the same study, making it difficult to provide evidence of one example of the purity of nurse dose. Several researchers have examined education and experience or skill mix in relation to various outcomes. We posit that all three variables together (educational preparation, experience of nursing staff, and skill mix) may represent an increased concentration of nursing knowledge, which can be applied to improve the quality of patient care. However, further research will be needed to test our assertion.

\section{Nurse Dose: Amount}

Another critical attribute of nurse dose is the number of nurses. We identified one empirical indicator for the amount of nurse dose: the actual, not budgeted, number of full-time equivalent (FTE) nursing personnel who work on a particular unit. FTEs represent the total number of nurses available to provide care, resulting in the achievement of favorable outcomes. The continuing nursing shortage means that many nursing units may have unfilled vacancies. Consequently, using budgeted or unfilled FTEs to represent the amount of nurse dose would not offer a true picture of the actual number of nurses who are providing care to a group of patients.

Researchers have examined the effects of the amount of nursing care on patient outcomes using both $\mathrm{RN}$ and licensed practical nurse (LPN) FTEs (Mark, Harless, McCue, \& Xu, 2004; Unruh, 2003). Differences in the construction of the FTE variables may account for variation in findings. Unruh found an increase in pneumonia with more FTEs, whereas Mark and colleagues found a decrease in pneumonia, but only when the LPN FTEs were low (Mark, Harless, et al.; Unruh).

\section{Nurse Dose: Frequency}

The frequency of nurse dose refers to the number of times during a shift that nursing personnel interact with patients, increasing the opportunity for a patient to receive nursing care. Two staffing variables serve as empirical indicators for nurse dose frequency: hours per patient day (HPPD) and the nurse-patient ratio. In general, HPPD refers to the number of hours of paid nurse time relative to the number of patient days (Finkler \& Kovner, 2000). The nurse-patient ratio refers to the number of patients who are assigned to any one nurse on a nursing unit for a particular shift. HPPD and nurse-patient ratio can both serve as indicators of nurse dose frequency because how often a nurse interacts with a patient may be a function of the number of nurses who are available for patient care when needed, as well as the number of patients who require care from each nurse. While increasing HPPD and decreasing nurse-patient ratios do not guarantee more nurse-patient interactions, there is evidence that they might improve nurse surveillance and contribute to better patient outcomes (Clarke \& Aiken, 2003).

All nursing personnel are usually counted in HPPD, but researchers have divided HPPD according to nursing classification (Blegen \& Vaughn, 1998; Cho, Ketefian, Barkauskas, \& Smith, 2003; Needleman, Buerhaus, Mattke, Stewart, \& Zelevinsky, 2002). Although both Blegen and Vaughn, and Cho et al. constructed a variable of the ratio of RN-HPPD to total HPPD, their findings were contradictory. Blegen and Vaughn used the nursing unit as the level of analysis and conducted their study in one hospital; whereas Cho et al. used a California database and studied relationships at the hospital level. The extent to which either level of analysis or data source may have affected the relationships is unknown. Differing levels of analysis may influence observed relationships between attributes of nurse dose and outcome variables.

Interestingly, very few researchers have investigated nurse-patient ratios and their relationship to outcomes. Part of the reason may be because nurse-patient ratios vary from unit to unit, and the majority of staffing studies have been conducted at the hospital level. Aiken, Clarke, Sloane, Sochalski, and Silber (2002) demonstrated that increases in nurse-patient ratios, defined as the "mean patient load across all staff registered nurses" (p. 1988), were associated with an increased likelihood of mortality and failure to rescue for hospitalized patients. Kovner, Jones, 
Zhan, Gergen, and Basu (2002) constructed a variable from the number of full-time equivalent RNs as a proportion of patient days, and found an inverse relationship with pneumonia, but not with three other adverse events (deep vein thrombosis, pulmonary events, urinary tract infections).

\section{Nurse Dose: Duration}

Duration is the fourth critical attribute of nurse dose, and can be reflected by patients' length of stay (LOS). LOS, as an empirical indicator of the duration of nurse dose, represents the total period during which patients are cared for by nurses and hence exposed to nurse dose. Our definition is consistent with the one of duration that we found when conducting the concept analysis of dose. This empirical indicator of duration is the only one that is not derived from a staffing variable. It is based on observations that the decision to discharge a patient or prolong hospitalization is often based on whether or not the patient continues to require 24 hours nursing care, because nursing care in many instances is the primary clinical intervention for hospitalized patients (Aiken, Sochalski, \& Lake, 1997).

Although this observation suggests a relationship between duration, an indicator of nurse dose, and outcomes, the nature of the association between nursing care and LOS is not clear due to lack of relevant research. A LOS that is too short or too long may have negative consequences. Short LOS may limit the provision of required care, while prolonged hospitalization may be associated with iatrogenic complications, as well as increased morbidity and mortality (Carey, Sheth, \& Braithwaite, 2005). Thus, the relationship between duration and patient outcomes requires further investigation.

The review of staffing research used in the concept derivation process revealed that no single study examined the proposed critical attributes of nurse dose altogether or in combination. This limited our ability to determine the simultaneous utility of the attributes and corresponding empirical indicators in operationalizing the concept of nurse dose in its entirety. The lack of a theoretically coherent approach across staffing studies reviewed suggested that our version of nurse dose could fill a void in this area of research.

\section{DISCUSSION}

Nurse dose is a concept proposed to underpin staffing research. Concept derivation led to a reconceptualization of nurse dose as a unified entity, defined as the level of nursing required to produce the intended quality outcomes and operationalized in empirical indicators reflected by relevant variables used in staffing studies. In its entirety, nurse dose encompasses all of the critical attributes that need to be considered in prescribing the treatment of nursing care, which has not been part of staffing research to date. Nurse dose allows for assessing nursing care as a treatment. As in medicine, where prescribing a dose of a medication by only specifying the frequency of the drug is insufficient, the same applies to the prescription of the level of nursing required to achieve favorable outcomes.

The re-conceptualization of staffing variables as indicators of nurse dose offers a useful alternative to pull staffing variables together, in a meaningful way, under a unified concept. Multivariate statistical techniques (such as structural equation modeling) could be used to examine the interrelations among empirical indicators of nurse dose and between these indicators and outcomes.

The identified critical attributes and empirical indicators of nurse dose could guide efforts at standardizing the definition and measurement of staffing variables. Standardization is critical to comparison and interpretation of findings across studies, which is important for building knowledge in the field. If future researchers use all of the critical attributes identified in this article, in effect considering the impact of nurse dose as a unified entity on a patient outcome, similarities will be seen more easily across those studies, interpretation will be easier, and advancements in understanding nursing's impact on patient outcomes will emerge.

The conceptualization of staffing variables as distinct but interrelated empirical indicators of nurse dose implies the assessment of more than one variable in any given study, which enhances construct validity; it also demands attention to examining the independent and combined effects of staffing variables on outcomes. This in turn, will inform us of which critical attribute(s) and empirical indicator(s) of nurse dose contribute(s) most to the achievement of beneficial outcomes. For example, Mark, Harless, et al. (2004) found that pneumonia rates decreased with a higher proportion of RN FTEs, but only when licensed practical nurse FTEs were low. These findings are easier to interpret when thought of in nurse dose terms. It may be that for the adverse outcome of pneumonia, the purity of nurse dose (education, experience, and skill mix) is more critical than the amount of the dose (FTEs). But without including 
all critical attributes and empirical indicators of nurse dose, future researchers would not be able to answer the question of which attribute(s) with corresponding indicators of nurse dose is (are) more likely to reduce the incidence of pneumonia.

Knowledge of attributes' and indicators' contributions to outcomes is a valuable tool for nurse administrators to help them make evidence-based staffing decisions. By thinking of various staffing variables as empirical indicators of nurse dose, nurse administrators may be able to base staffing decisions on evidence. By combining identified indicators into the critical attributes of nurse dose as described here, nurse administrators may turn nurse dose into a prescriptive tool that promotes better patient outcomes. When describing the impact of nursing on patient outcomes, nurse administrators may find that referring to nursing care in nurse dose terms is better understood by other hospital administrators.

Although we posit that higher nurse doses should be associated with greater improvements in patient outcomes, we acknowledge that there is probably some point above which a higher nurse dose would have no further impact on outcomes. Researchers have already shown that the relationship between hours of nursing time spent on care and patient acuity is non-linear (Blegen, Goode, \& Reed, 1998), and that there are levels of RN staffing beyond which further increases may no longer decrease patient mortality (Mark, Harless, et al., 2004). Determining the optimal nurse dose for any patient outcome will require ongoing investigation.

\section{CONCLUSION}

In this article, we fill a gap in nursing literature by providing a conceptualization and operationalization of nurse dose as captured by variables used in staffing studies. Our conceptualization proposes that (a) nurse dose is a unified concept reflected in the four critical attributes of purity, amount, frequency, and duration, and (b) optimum patient outcomes can be achieved by prescribing the correct nurse dose.

The work to clarify relationships among various critical attributes of nurse dose lies ahead of us. We also must confirm the nature of the association of the empirical indicators to nurse dose and to each other. Our next step will be to validate the empirical indicators of nurse dose and to examine their linkage to outcomes. We invite our colleagues to comment on our efforts, recognizing that through dialogue and discourse we all contribute to moving our discipline forward.

\section{REFERENCES}

American Academy of Colleges of Nursing. (2001). Position statement: The baccalaureate degree in nursing as minimal preparation for professional practice. Journal of Professional Nursing, 17, 267269.

Aiken, L.H., Clarke, S.P., Cheung, R.B., Sloane, D.M., \& Silber, J.H. (2003). Educational levels of hospital nurses and surgical patient mortality. JAMA, 290, 1617-1623.

Aiken, L.H., Clarke, S.P., Sloane, D.M., Sochalski, J., \& Silber, J.H. (2002). Hospital nurse staffing and patient mortality, nurse burnout, and job dissatisfaction. JAMA, 288, 1987-1993.

Aiken, L.H., Sochalski, J., \& Lake, E.T. (1997). Studying outcomes of organizational change in health services. Medical Care, 35, NS6-NS18.

Blegen, M.A., Goode, C.J., \& Reed, L. (1998). Nurse staffing and patient outcomes. Nursing Research, 47, 43-50.

Blegen, M.A., \& Vaughn, T. (1998). A multisite study of nurse staffing and patient occurrences. Nursing Economics, 16, 196-203.

Blegen, M.A., Vaughn, T.E., \& Goode, C. (2001). Nurse experience and education: Effect on quality of care. Journal of Nursing Administration, 31, 33-39.

Borelli, B., Sepwinall, D., Ernst, D., Bellg, A.J., Czajkowski, S., Breger, R., et al. (2005). A new tool to assess treatment fidelity and evaluation of treatment fidelity across 10 years of health behavior research. Journal of Consulting \& Clinical Psychology, 73, 852-860.

Boyce, C.A., Brow, M.B., Cote, K.C., DeSisto, M.C., Evans, D.A., Gorman, D., et al. (2001). End the debate: Entry level into practice should be the master's degree. Journal of Nursing Administration, 31, 166-168.

Brooten, D., \& Naylor, M.D. (1995). Nurses' effect on changing patient outcomes. Journal of Nursing Scholarship, 27, 95-99.

Brooten, D., \& Youngblut, J.M. (2006). Nurse dose as a concept. Journal of Nursing Scholarship, 38, 94-99.

Carey, M.R., Sheth, H., \& Braithwaite, R.S. (2005). A prospective study of reasons for prolonged hospitalizations on a general medicine teaching service. Journal of General Internal Medicine, 20, 108115.

Cho, S.H., Ketefian, S., Barkauskas, V.H., \& Smith, D.G. (2003). The effects of nurse staffing on adverse events, morbidity, mortality, and medical costs. Nursing Research, 52, 71-79.

Clarke, S.P., \& Aiken, L.H. (2003). Failure to rescue. American Journal of Nursing, 103(1), 42-47.

Condelli, W.S., \& Hubbard, R.L. (1994). Relationship between time spent in treatment and client outcomes from therapeutic communities. Journal of Substance Abuse Treatment, 11, 25-33. 
Estabrooks, C.A., Midodzi, W.K., Cummings, G.G., Ricker, K.L., \& Giovanetti, P. (2005). The impact of hospital nursing characteristics on 30-day mortality. Nursing Research, 54, 74-84.

Finkler, S.A., \& Kovner, C.T. (2000). Financial management for nurse managers and executives. (2nd ed.). Philadelphia: Saunders.

Flay, B.R., \& Allred, C.G. (2003). Long-term effects of the Positive Action Program. American Journal of Health Behavior, 27, S6-S21.

Forgatch, M.S., Patterson, G.R., \& DeGarmo, D.S. (2005). Evaluating fidelity: Predictive validity for a measure of component adherence to the Oregon Model of Parent Management training. Behavior Therapy, 36, 3-13.

Jiang, H.J., Stocks, C., \& Wong, C.J. (2006). Disparities between two common data sources on hospital nurse staffing. Journal of Nursing Scholarship, 38, 187193.

Kovner, C.T., Jones, C., Zhan, C., Gergen, P.J., \& Basu, J. (2002). Nurse staffing and postsurgical adverse events: An analysis of administrative data from a sample of U.S. hospitals, 1990-1996. Health Services Research, 37, 611-629.

Lichtig, L.K., Knauf, R.A., \& Milholland, D.K. (1999). Some impacts of nursing on acute care hospital outcomes. Journal of Nursing Administration, 29, 25-33.

Lipsey, M.W. (1990). Design sensitivity. Statistical power for experimental research. Thousand Oaks, CA: Sage.

Mark, B.A., Harless, D.W., McCue, M., \& Xu, Y. (2004). A longitudinal examination of hospital registered nurse staffing and quality of care. Health Services Research, 39, 279-300.

Mark, B.A., Hughes, L.C., \& Jones, C.B. (2004). The role of theory in improving patient safety and quality health care. Nursing Outlook, 52, 1116.
Needleman, J., Buerhaus, P.I., Mattke, S., Stewart, M., \& Zelevinsky, K. (2002). Nurse-staffing levels and the quality of care in hospitals. New England Journal of Medicine, 346, 1715-1722.

Person, S.D., Allison, J.J., Kiefe, C.I., Weaver, M.T., Williams, O.D., Centor, R.M., et al. (2004). Nurse staffing and mortality for medicare patients with acute myocardial infarction. Medical Care, 42, 4-12.

Peterson, M.D., Rhea, M.R., \& Alvar, B.A. (2004). Maximizing strength development in athletes: A meta-analysis to determine the dose-response relationship. Journal of Strength \& Conditioning Research, 18, 377-382.

Rhea, M.R., Alvar, B.A., Burkett, L.N., \& Ball, S.D. (2003). A meta-analysis to determine the dose response for strength development. Medicine \& Science in Sports \& Exercise, 35, 456-464.

Scott, A.G., \& Sechrest, L. (1989). Strength of theory and theory of strength. Evaluation \& Program Planning, 12, 329-336.

Sidani, S., \& Braden, C.J. (1998). Evaluating nursing interventions. A theory-driven perspective. Thousand Oaks, CA: Sage.

Simpkins Chaput, S., Little, P.M.D., \& Weiss, H. (2004). Understanding and measuring attendance in out-ofschool time programs. Harvard Family Research Project. http://www.gse.harvard.educ/hfrp/projects/ afterchool/resources/issuebrief7.html

Unruh, L. (2003). Licensed nurse staffing and adverse events in hospitals. Medical Care, 41, 142-152.

Verran, J.A. (1997). The value of theory-driven (rather than problem-driven) research. Seminars for Nurse Managers, 5, 169-172.

Walker, L.O., \& Avant, K.C. (2005). Strategies for theory construction in nursing. Upper Saddle River, NJ: Pearson Education, Inc.

Yeaton, W.H. (1985). Using measures of treatment strength and integrity in planning research. New Directions for Program Evaluation, 27, 49-62. 